Voix et Images

voixetimages

\title{
L’écriture de la vie pensée
}

\section{Jacques Paquin}

Volume 20, numéro 2 (59), hiver 1995

Archéologie du littéraire au Québec

URI : https://id.erudit.org/iderudit/201175ar

DOI : https://doi.org/10.7202/201175ar

Aller au sommaire du numéro

Éditeur(s)

Université du Québec à Montréal

ISSN

0318-9201 (imprimé)

1705-933X (numérique)

Découvrir la revue

Citer cet article

Paquin, J. (1995). L'écriture de la vie pensée. Voix et Images, 20(2), 459-463.

https://doi.org/10.7202/201175ar

Ce document est protégé par la loi sur le droit d'auteur. L'utilisation des services d'Érudit (y compris la reproduction) est assujettie à sa politique d'utilisation que vous pouvez consulter en ligne.

https://apropos.erudit.org/fr/usagers/politique-dutilisation/ 


\section{L'écriture de la vie pensée}

Jacques Paquin, Université de Regina

Je n'amorcerai pas ce commentaire critique en faisant le compte et le mécompte des études consacrées à l'essai. D'ailleurs, peut-ón exactement savoir de quoi il en retourne lorsqu'on voit regroupés sous 
cette étiquette des écrits aussi différents dans leur démarche que le journal intime et le pamphlet? Et que dire des textes qui prennent pour objet l'histoire ou même un objet scientifique? Sans mentionner que certains auteurs ont commencé depuis peu à apposer leurs propres étiquettes, comme celui d'“ essais-fictions ". D'autre part, la liste des "essais littéraires" de telle maison d'édition nous éclaire-elle pour autant sur les caractéristiques du genre? Encore faudrait-il comprendre ce que recouvre (ou dévoile) l'épithète "littéraire". Doit-on déduire que l'essai est la chasse gardée des seuls littérateurs? Certes non, bien que les essayistes, dans leur grande majorité, soient des universitaires. Mais voilà un genre qu'il faut éviter d'identifier exclusivement avec la "littérature d'idées", puisqu'à sa manière, il participe d'une écriture. Le défi demeure donc: comment parler de l'essai sans s'enliser dans un débat d'idées?

Robert Vigneault a relevé le gant en livrant le fruit de quelque vingt années de recherches et d'enseignement consacrées à l'essai ${ }^{1}$. D'entrée de jeu, le chercheur dévoile sa position épistémologique : "Je n'ai pas procédé a priori, c'est-à-dire à partir d'une sorte de modèle construit de l'essai, que j'aurais ensuite appliqué comme une grille aux textes" (p. 15). En effet, bien que maîtrisant parfaitement les enjeux théoriques de son objet d'investigation, Robert Vigneault éprouve une certaine méfiance à l'égard de l'outillage sophistiqué des théories lorsque celles-ci exercent un pouvoir trop exclusif sur le déroulement de l'analyse. En cela, sa pensée est tributaire d'une formation humaniste qui influe sur maints aspects de son approche du texte littéraire, comme on le verra plus loin. L'ouvrage comprend deux parties, la première intitulée "Réflexion théorique", la seconde "Essais spéculaires". Mais la théorie n'a pas précédé la pratique des textes, bien au contraire, puisque l'auteur avoue avoir voulu " procéder inductivement vers une "théorie" qui ne fût pas autre chose que la "réflexion" des observations consignées dans les essais critiques de la deuxième partie" (p. 15). Essayistes du $\mathrm{XIX}^{\mathrm{e}}$ siècle et auteurs féminins en sont absents toutefois, ce qui précise, tout en la limitant, la portée de son propos. On devine que l'auteur s'est surtout laissé guider par ses goûts personnels.

La première partie, qui constitue le volet théorique de l'ouvrage, propose une définition comprenant les grands enjeux formels de l'écriture de l'essai : "discours argumenté d'un SUJET énonciateur qui interroge et s'approprie le vécu par et dans le langage" (p. 21). Cet énoncé forme en quelque sorte la matrice de l'ensemble et sera par la suite analysé dans le détail à partir de ses grandes composantes. Du point de vue générique, l'essai ne peut être considéré un génre à part entière que si on évite de l'enfermer dans la catégorie anglo-saxonne 
de non fiction. À ce propos, cependant, il aurait été intéressant de confronter cette opinion avec les travaux de Genette qui s'inspire en partie de cette taxinomie ${ }^{2}$. L'essai serait, au contraire, un discoúrs fictif dans la mesure où il est le produit d'un agencement d'idées, qu'il "invente littérairement la vie pensée de l'homme" (p. 22). On parlera donc de "fiction idéelle" (p. 37), formulation que l'auteur emprunte à André Belleau. Cette appellation possède en outre l'avantage d'établir la distinction entre essai et discours scientifique. Par ailleurs, contrairement aux autres genres canoniques (roman, poésie, théâtre), l'essai ne recourt pas aussi systématiquement aux médiations symboliques. Cela s'explique par le fait que le genre constitue une parole dont le cheminement s'articule selon l'immédiateté de l'expérience vécue. L'essai interroge le flux mouvant de la vie pour atteindre la vie en soi plutôt que la vie concrète, la vie plutôt que la vie selon les termes de Lukács, que Vigneault reformule de manière plus opératoire: "Exigeante quête de substance, l'essai prend forme en soumettant la contingence du vécu à la haute exemplarité des idées (valeurs)" (p. 38-39).

Lukács, Bachelard, Adorno et, dans une moindre mesure, Montaigne, sont ensuite conviés comme des modèles du genre. Le choix pourra paraître discutable, mais pour Vigneault, ces lectures électives valent pour les interrogations qu'elles ont pu susciter, peu importe la place que leur attribue l'histoire littéraire. Lukács a eu le mérite de présenter l'essai "comme un phare pour. les autres formes d'art, il permet d'en faire resplendir le sens" (p. 53). Quant à Bachelard, il se montre essayiste lorsqu'il délaisse la méthode scientifique pour l'approche phénoménologique, donnant ainsi libre cours au "discours d'un sujet pour qui le réel [...] n'existe que comme expérience traduite dans le langage d'un rêveur de mots" (p. 58). Les convictions idéologiques d'Adorno obligent cependant le critique à les confronter à ses propres présupposés philosophiques, ce qui dénote chez l'auteur un souci évident d'accueillir la pensée de l'autre malgré d'évidentes réserves. Sont ensuite réunis avec un bonheur inattendu Lukács et Bachelard dans une même communauté d'écriture, alors que les démarches diamétralement opposées d'Adorno et de l'essayiste hongrois donnent lieu à un débat captivant au cours duquel Vigneault prend le parti de Lukács. L'ạllusion à Montaigne demeure toutefois timide et semble plutôt prétexte à retracer l'histoire et l'étymologie du vocable "essai". Vu le propos, cet aspect de la question aurait dû apparaître beaucoup plus tôt dans l'ouvrage. La première partie se clôt sur un "projet de typologie: les registres de l'essai”. Plutôt que de miser sur la rectitude terminologique, toujours sujette à remaniements, on a préféré parler en termes de registres: polémique, introspectif, 
cognitif et absolu. Le registre polémique donne lieu à une confrontation entre les positions de Vigneault et les théories de Marc Angenot sur le pamphlet ${ }^{3}$. Moins familiers que les trois précédents, mais aussi, à mon avis, plus difficilement conceptualisables, les "essais absolus" (Robert Vigneault cite Joseph Bonenfant) "traitent un sujet hyperonymique qui serait avant tout la vie; le portrait de soi n'est plus prioritaire" (p. 97). Malgré son grand pouvoir suggestif, le recours à un vocable aussi indéfinissable que "la vie " ouvre la voie à une multitude d'interprétations. Est-ce que Baudelaire n'avait pas de son côté formulé une distinction similaire entre l'éphémère et l'éternel, ce dernier terme provenant d'une conception on ne peut plus classique? Une telle démarche laissera insatisfait le théoricien soucieux de manier des notions dénuées de toute ambiguité. Il y a un hiatus évident entre une pensée nourrie d'humanisme et la réflexion théorique.

La seconde partie s'ouvre sur l'œuvre de Pierre Vadeboncœur. Elle constitue sans nul doute la pièce de résistance de cet essai critique. Robert Vigneault se sent tout à fait à l'aise dans les "parcours idéels" d'un écrivain qui, fait exceptionnel dans nos lettres, est devenu essayiste au fil de l'écriture. De manière admirable, ces cinq essais dégagent la vision du monde marquée du sceau d'une pensée duelle, partagée entre "le je de l'écriture polémique, pamphlétaire, persuasive, d'une part, et le je intime de l'écriture du sujet" (p. 154). Tout aussi importante, à mon avis, est la nature des liens qui s'établissent entre le critique et l'œuvre de l'essayiste. Il s'agit à proprement parler d'une rencontre entre un théoricien et une œuvre. Sans jamais renoncer à sa subjectivité, le chercheur établit avec l'œuvre de Vadeboncœur une relation dialogique qui sait même s'accommoder de réticences. Par la manière dont lui-même aborde les œuvres, Robert Vigneault montre, par l'exemple, ce qu'il entend par essai critique, "où le je de l'écriture ne réussit à s'implanter dans son propre texte que dans un véritable corps à corps avec le texte d'autrui " (p. 227). La composition du chapitre médian m'a quelque peu déconcerté, du fait que s'enchaînent une analyse fouillée d'un échantillon représentatif des écrits de Cité libre et un exposé laudatif sur la pensée d'André Belleau.

"Les essais critiques sur l'écriture" du dernier chapitre sont une compilation d'articles critiques sur des essayistes ou des théoriciens du genre. Figurent entre autres les noms de Jean-Louis Major, Philippe Haeck, Frânçois Ricard, Jacques Godbout, Marc Angenot et Laurent Mailhot. L'unité est maintenue grâce à la finesse des analyses et à la grande sensibilité esthétique de l'auteur. Mais tandis que la première partie constituait un exercice rigoureux sur la nature de l'essai, il arrive que l'analyste se laisse aller à la critique de contenu, comme si le théo- 
ricien cédait à nouveau la parole au philosophe. L'auteur lui-même s'est diverti à écrire, en guise d'épilogue, un essai fantaisiste où il fait part notamment de ses observations sur l'enseignement de la littérature à l'université. Que Robert Vigneault se rassure sur ce point: il fait déjà œuvre d'essayiste tout au long de son ouvrage, ne serait-ce que par ses réflexions inspirées par la "passion du sens". Mais surtout, il apporte une contribution remarquable aux (trop rares) études sur l'essai.

1. Robert Vigneault, L'Écriture de l'essai, Montréal, l'Hexagone, coll. «Essais littéraires., 1994, $330 \mathrm{p}$.

2. Gérard Genette, Fiction et Diction, Paris, Seuil, coll. *Poétique *, 1991, 151 p.

3. Marc Angenot, La Parole pamplétaire. Typologie des discours modernes, Paris, Payot, coll. «Langages et sociétés *, 1982, 425 p. 